hep-ph/0112086

December 2001

\title{
MSSM Constraints from Higgs Boson Searches
}

\section{André Sopczak}

Lancaster University

\begin{abstract}
The LEP era has brought immense progress in searches for Higgs bosons over the last 12 years which will guide searches at future colliders. The evolution of the Higgs boson mass limits is reviewed with the focus on results from general parameter scans in the Minimal Supersymmetric extension of the Standard Model (MSSM) in contrast to the so-called benchmark limits. The hint for a Standard Model (SM) Higgs boson of $115.6 \mathrm{GeV}$ can also be interpreted as a preference for a Higgs boson of that mass in the MSSM. Further small data excesses allow the hypothesis that the neutral Higgs bosons of the MSSM all have masses between 90 and $116 \mathrm{GeV}$.
\end{abstract}

Presented at the Seventh Topical Seminar on the Legacy of LEP and SLC, Siena, Italy, October 2001 



\title{
MSSM Constraints from Higgs Boson Searches
}

\author{
André Sopczak, Lancaster University
}

The LEP era has brought immense progress in searches for Higgs bosons over the last 12 years which will guide searches at future colliders. The evolution of the Higgs boson mass limits is reviewed with the focus on results from general parameter scans in the Minimal Supersymmetric extension of the Standard Model (MSSM) in contrast to the so-called benchmark limits. The hint for a Standard Model (SM) Higgs boson of $115.6 \mathrm{GeV}$ can also be interpreted as a preference for a Higgs boson of that mass in the MSSM. Further small data excesses allow the hypothesis that the neutral Higgs bosons of the MSSM all have masses between 90 and $116 \mathrm{GeV}$.

\section{Introduction}

The search for Higgs bosons has been one of the most important lines of research during the LEP era. Final LEP-1 results from data taken at the Z-resonance [1] and preliminary LEP-2 results for complete data up to $209 \mathrm{GeV}$ center-of-mass energy [2] were recently reviewed. The MSSM is well motivated and the most discussed extension of the SM. It predicts three neutral (h, H, and A) and two charged Higgs bosons. At the beginning of LEP operation in 1989, no radiative corrections to the neutral Higgs boson masses were calculated and $m_{\mathrm{h}}<m_{\mathrm{Z}}$ was predicted. First- and secondorder corrections increased the upper mass bound to about $130 \mathrm{GeV}$. The neutral Higgs boson masses and production cross sections strongly depend on various model parameters. At LEP two ways were chosen to present the mass limits: benchmark limits for a certain set of MSSM parameters and mass limits based on MSSM parameter scans. Details of the benchmark and scan parameters are given for example in Refs. [3, 4 .

\section{Largely reduced $\mathrm{h}$ mass limit}

The importance of a MSSM parameter scan to set mass limits was already realized at LEP-1. The region marked by the thick black line in Fig. 1 shows that the $\mathrm{h}$ mass limit is reduced from 41 to $25 \mathrm{GeV}$ in a parameter scan compared to benchmark results [5, 6.

\section{Removal of A mass limit}

An early LEP-2 study showed that a benchmark limit of $52 \mathrm{GeV}$ on the A mass [7] disappeared completely in a MSSM parameter scan [8] (Fig. 2).

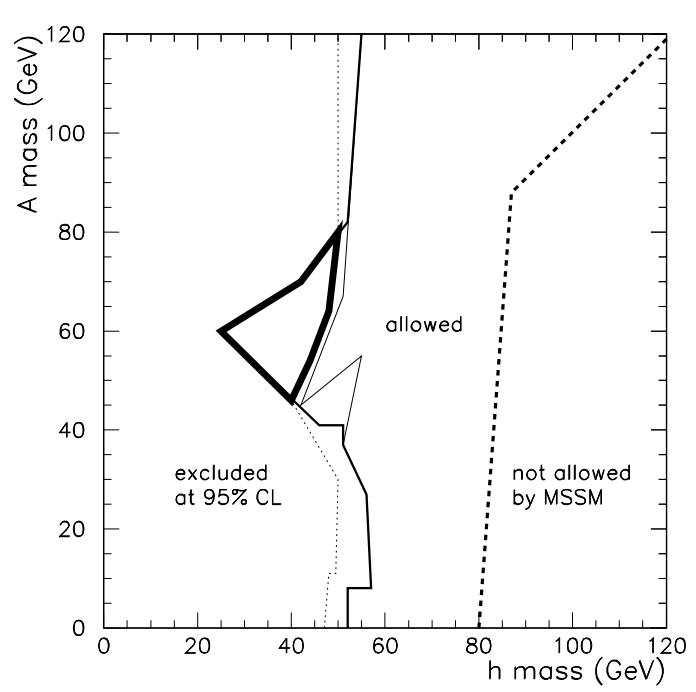

Figure 1. $91 \mathrm{GeV}\left(m_{\mathrm{h}}, m_{\mathrm{A}}\right)$ result.

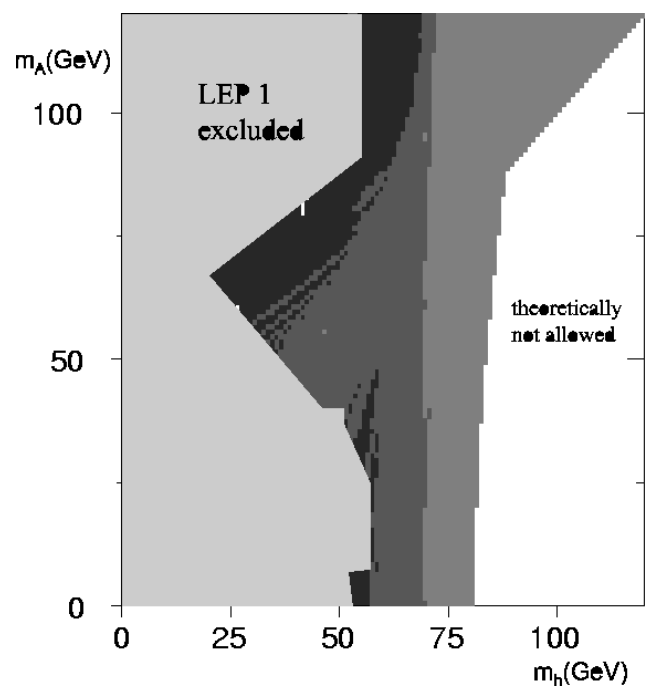

Figure 2. $172 \mathrm{GeV}\left(m_{\mathrm{h}}, m_{\mathrm{A}}\right)$ result. Only the dark region is excluded in addition to LEP-1. 


\section{Similar limits from different experiments}

The results from very different parameter scan methods of different LEP experiments agree well as shown in Figs. 3 and 1 for $189 \mathrm{GeV}$ data [9.10]. The shaded region in Fig. 3 is excluded by chargeand color-breaking (CCB) criteria 9].

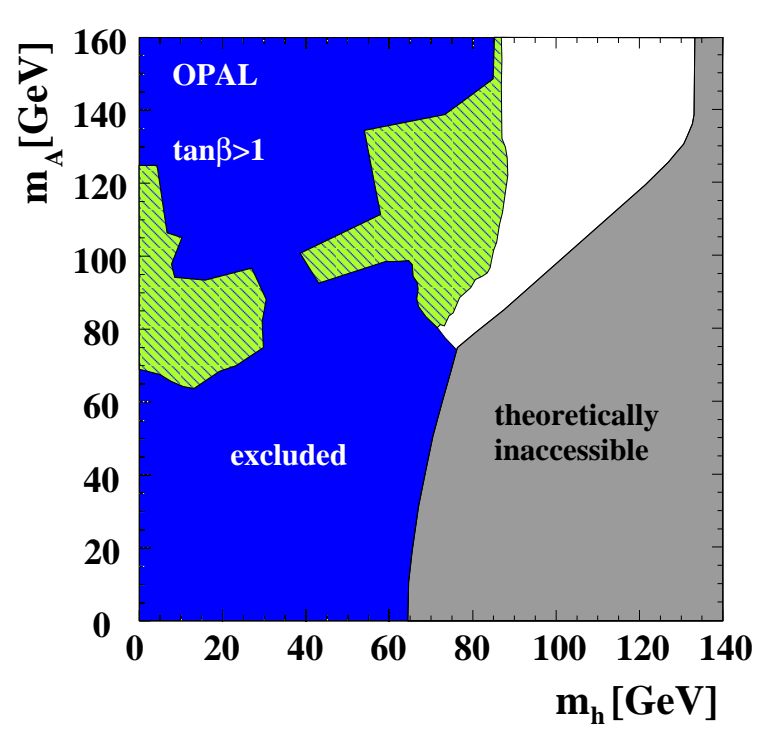

Figure 3. $189 \mathrm{GeV}\left(m_{\mathrm{h}}, m_{\mathrm{A}}\right)$ result.

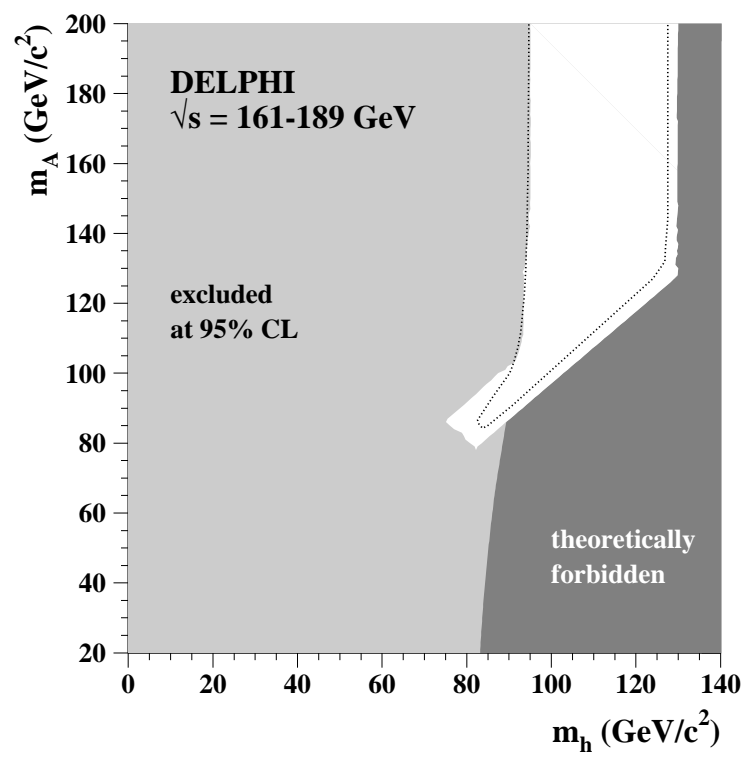

Figure 4. $189 \mathrm{GeV}\left(m_{\mathrm{h}}, m_{\mathrm{A}}\right)$ result. The dotted line indicates the corresponding benchmark limit.
Limits from charmless b-decays, the electroweak parameter $\Delta \rho$ and direct searches for Supersymmetric particles do not change the $\mathrm{h}$ and A mass limits [10]. The parameter scan reduced the benchmark limits by up to $7 \mathrm{GeV}$ 11, 10]. The constraints on $\tan \beta$ are given in Figs. 5 and 6 .

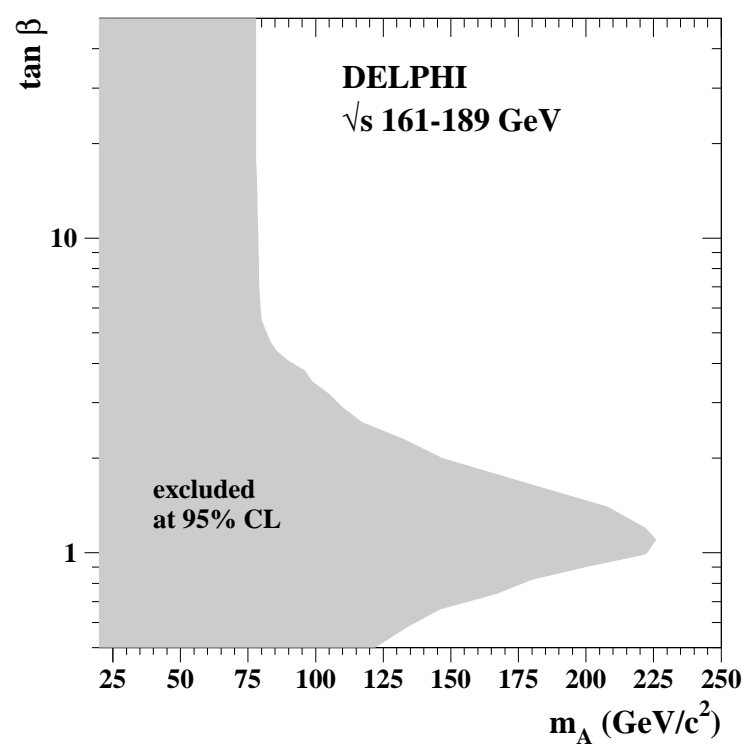

Figure 5. $189 \mathrm{GeV}\left(m_{\mathrm{A}}, \tan \beta\right)$ result.

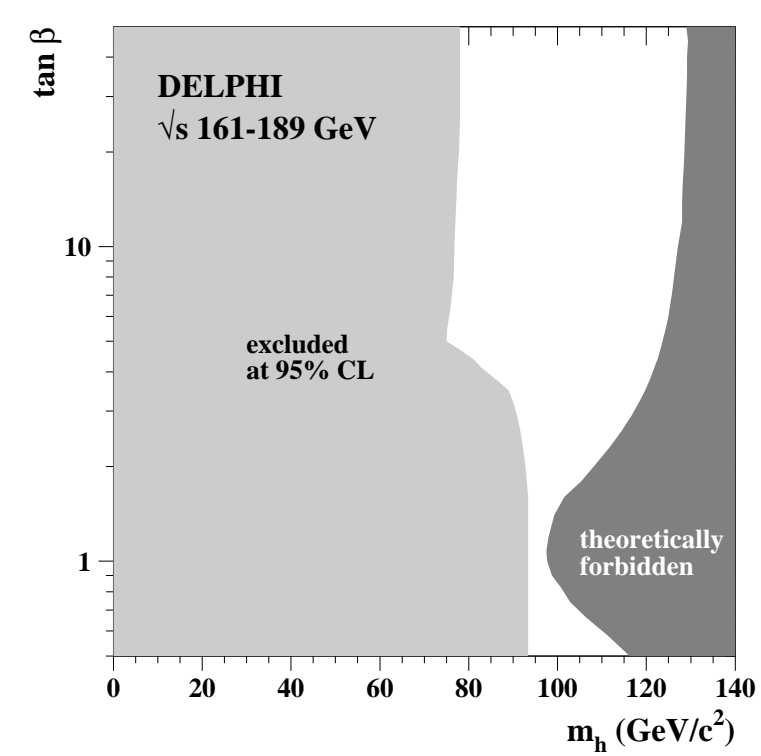

Figure 6. $189 \mathrm{GeV}\left(m_{\mathrm{h}}, \tan \beta\right)$ result. 


\section{Small reduction of limits}

With increasing center-of-mass energy, benchmark and parameter scan limits agree within $1 \mathrm{GeV}$ for $202 \mathrm{GeV}$ data 12 . The mass and $\tan \beta$ limits are given in Figs. 6, 8 and 9 .

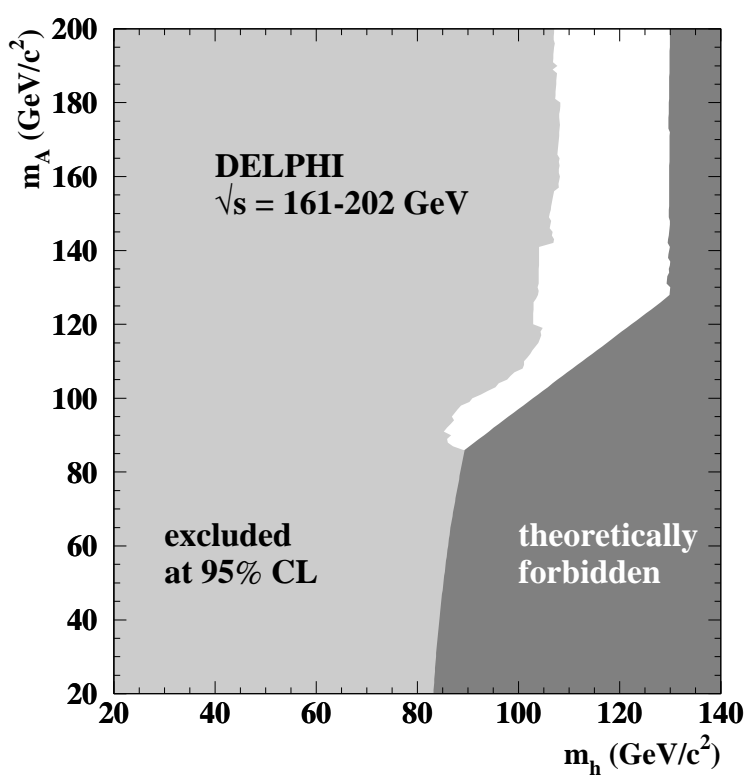

Figure 7. $202 \mathrm{GeV}\left(m_{\mathrm{h}}, m_{\mathrm{A}}\right)$ result.

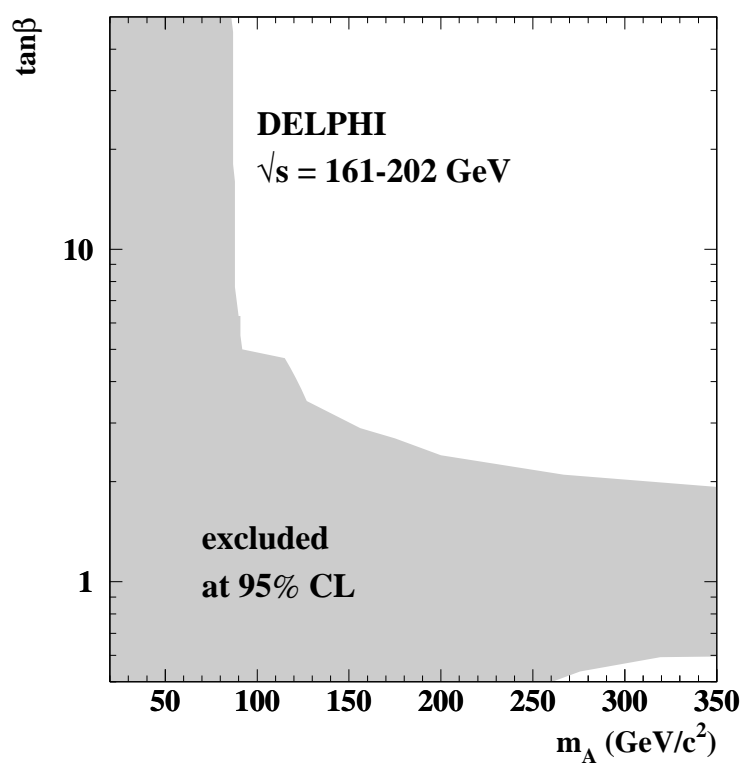

Figure 8. $202 \mathrm{GeV}\left(m_{\mathrm{A}}, \tan \beta\right)$ result.

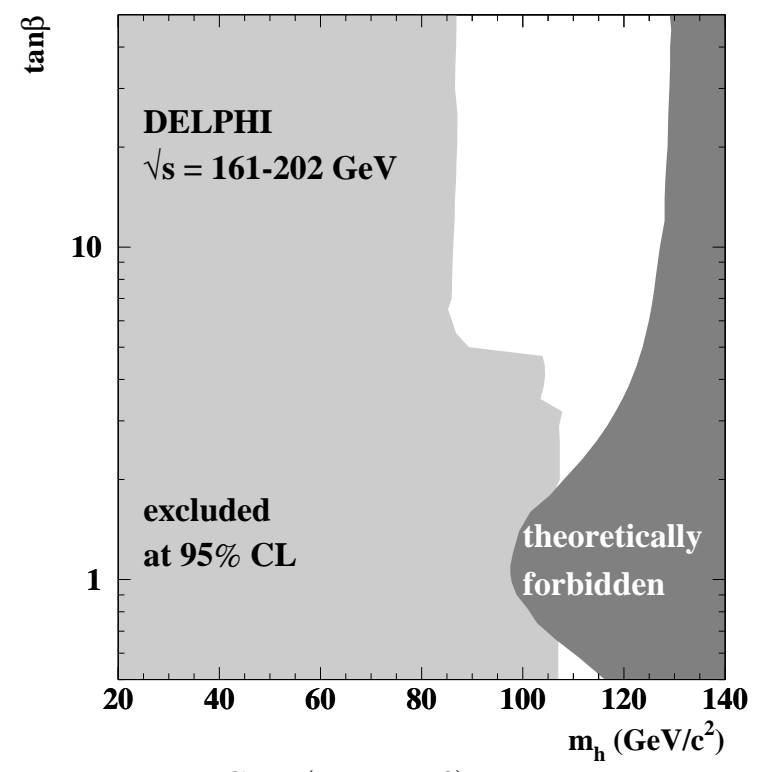

Figure 9. $202 \mathrm{GeV}\left(m_{\mathrm{h}}, \tan \beta\right)$ result.

\section{Combined LEP parameter scan limits}

The mass limits from benchmark and parameter scan agree within about $2 \mathrm{GeV}$ in the combination of the $202 \mathrm{GeV}$ data from all LEP experiments 13.14. The scan limits are given in Figs. 10, 11 and 12 .

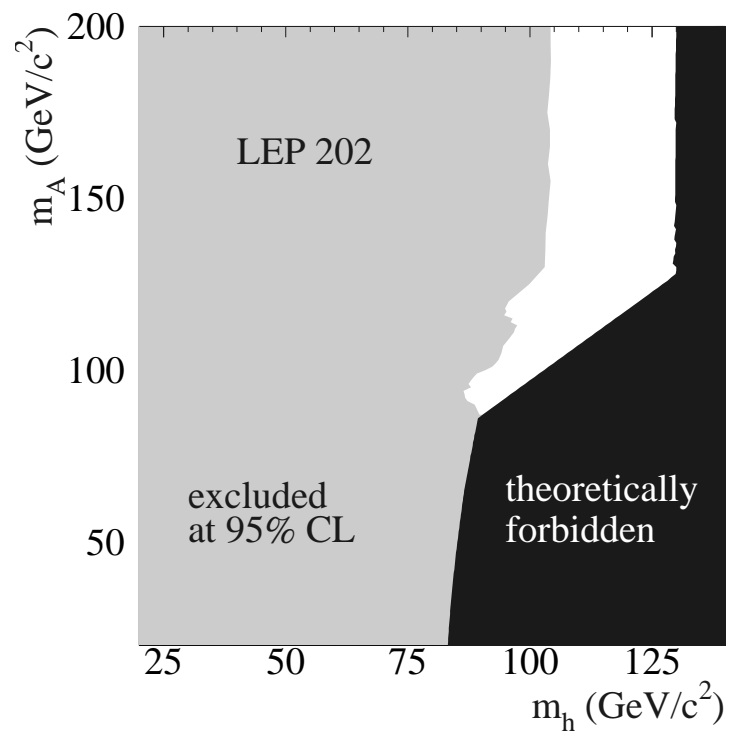

Figure 10. $202 \mathrm{GeV}\left(m_{\mathrm{h}}, m_{\mathrm{A}}\right)$ result. 


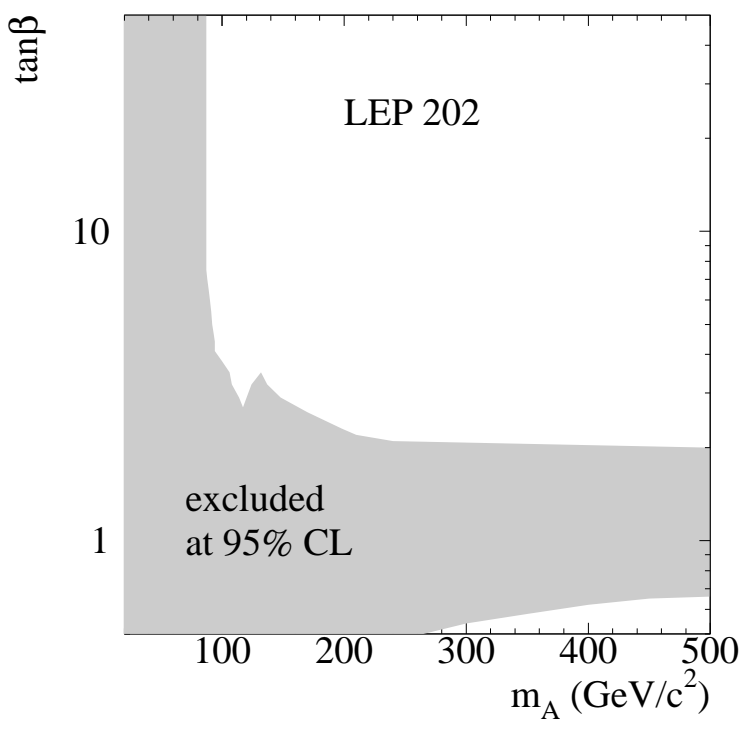

Figure 11. $202 \mathrm{GeV}\left(m_{\mathrm{A}}, \tan \beta\right)$ result.

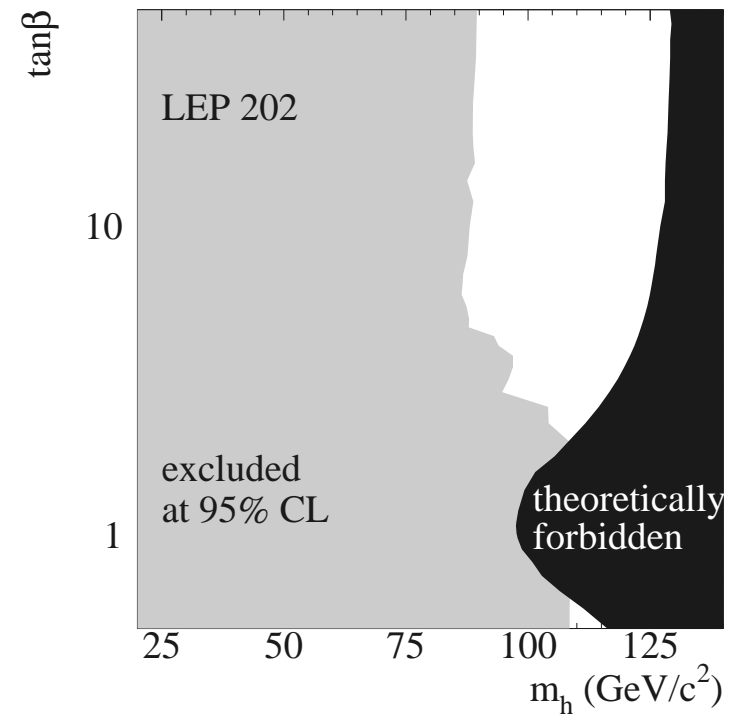

Figure 12. $202 \mathrm{GeV}\left(m_{\mathrm{h}}, \tan \beta\right)$ result.

\section{Latest results}

The latest benchmark and scan results including $209 \mathrm{GeV}$ data agree within $2 \mathrm{GeV}$ 15, 4 and give mass limits on $\mathrm{h}$ and $\mathrm{A}$ of $89 \mathrm{GeV}$ (Figs. 13, 14 and 15). The importance of a MSSM parameter scan is underlined in Fig. 16, which shows that large parameter regions exist where a Higgs boson could decay invisibly into a pair of neutralinos. Thus, only in conjunction with a dedicated search [16] the $\mathrm{h}$ and A mass limits are set.

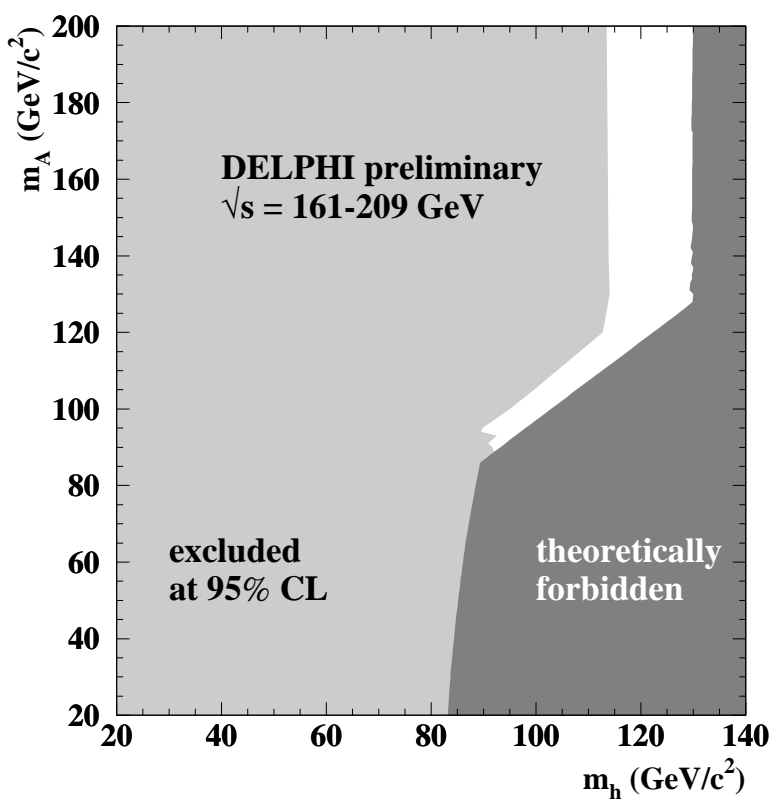

Figure 13. $209 \mathrm{GeV}\left(m_{\mathrm{h}}, m_{\mathrm{A}}\right)$ result.

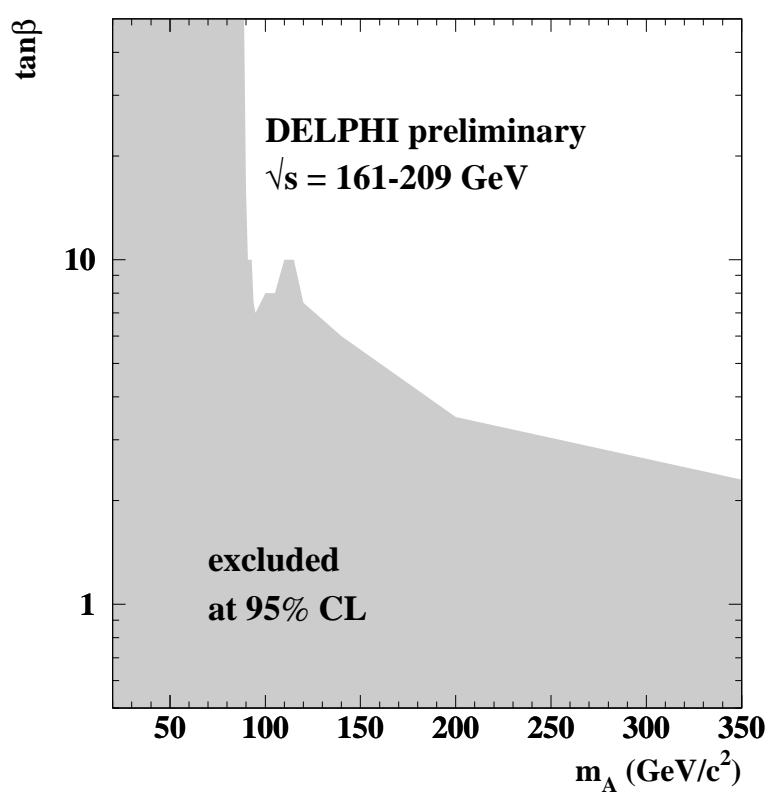

Figure 14. $209 \mathrm{GeV}\left(m_{\mathrm{A}}, \tan \beta\right)$ result. 


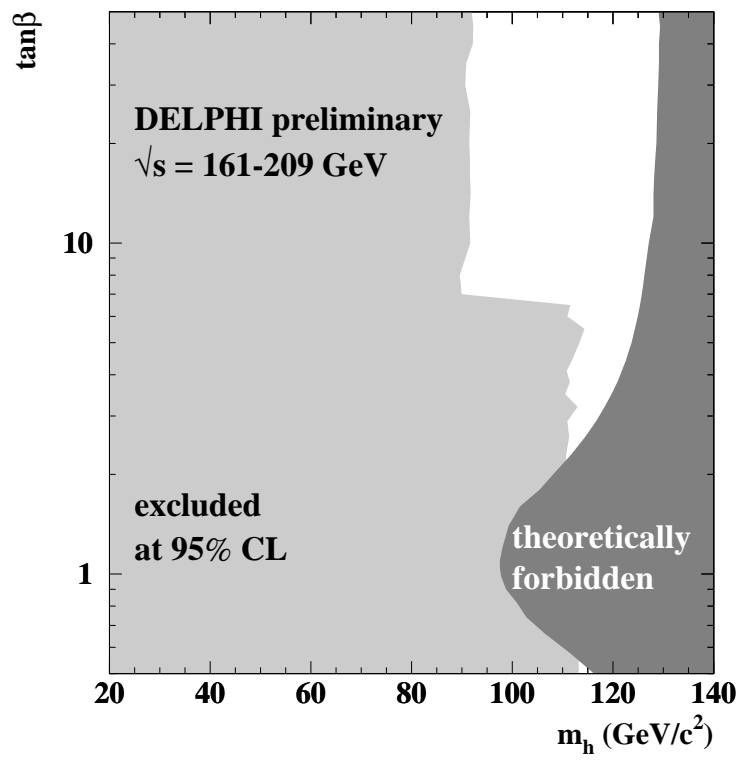

Figure 15. $209 \mathrm{GeV}\left(m_{\mathrm{h}}, \tan \beta\right)$ result.

\section{Three Higgs boson hypothesis}

For $202 \mathrm{GeV}$ data, taken in 1999, and first 2000 data, $\mathrm{h}$ and $\mathrm{A}$ mass limits were $2 \mathrm{GeV}$ below expectation [17]. This tendency is enhanced by including complete $209 \mathrm{GeV}$ data, in which case the limits are 3.1 to $3.6 \mathrm{GeV}$ below the expectations of about $95 \mathrm{GeV}$ [3]. A possible explanation is that the $\mathrm{HZ}$ excess at about $115 \mathrm{GeV}$ is due to the heavier scalar $\mathrm{H}$ and that, in addition, the production of hA with masses between 90 and $100 \mathrm{GeV}$ occurs [17]. Figure 17 (from [3]) shows a data excess above $2 \sigma$ for $m_{\mathrm{h}}+m_{\mathrm{A}}=187 \mathrm{GeV}$ in the b $\bar{b} b \bar{b}$ channel. The same data excess is also expressed by the confidence level $C L_{\mathrm{b}}$ for a signal observation as shown in Fig. 18 (from [3]). The hypothesis of the production of three MSSM Higgs bosons is supported by the data excess seen in Fig. 19 (from [18]) at $100 \mathrm{GeV}$ which could result from hZ production in addition to HZ production. For the reported MSSM parameters [17] $\cos ^{2}(\beta-\alpha) \approx 0.9$; therefore $\sin ^{2}(\beta-\alpha)=\xi^{2} \approx$ 0.1 . The $\xi^{2}$ limit in the $100 \mathrm{GeV}$ mass region shows a deviation of about $2 \sigma$ between the expected and observed limit, as seen in Fig. 20 (from 18]). Figure 21 shows that this new support is only observed in the complete LEP data.

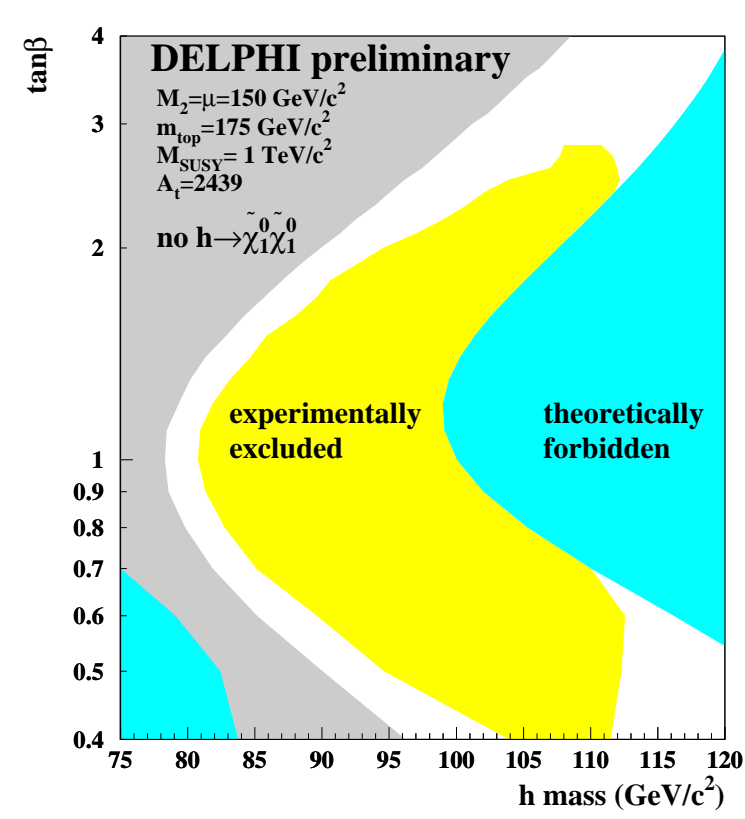

Figure 16. $209 \mathrm{GeV}\left(m_{\mathrm{h}}, \tan \beta\right)$ result from searches for invisibly decaying Higgs bosons.

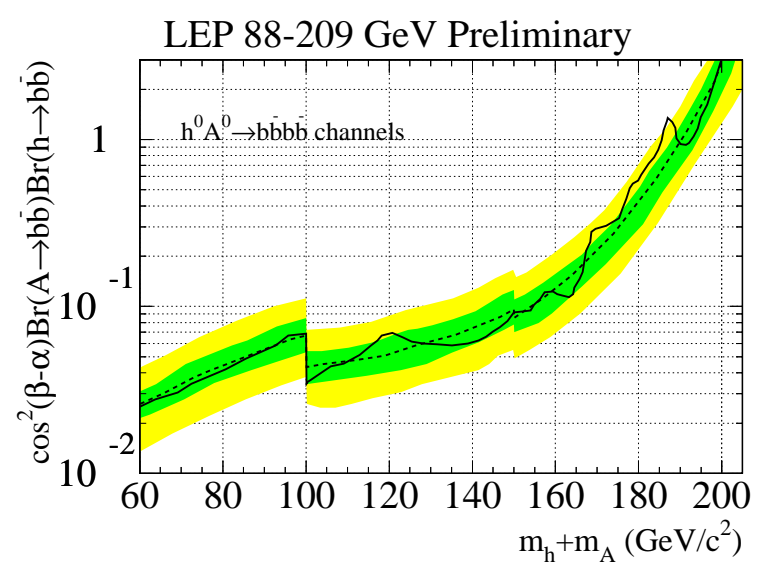

Figure 17. Limits on the hA cross section as a function of $m_{\mathrm{h}}+m_{\mathrm{A}}$ at $95 \%$ CL $\left(m_{\mathrm{h}} \approx m_{\mathrm{A}}\right)$ for the MSSM processes $\mathrm{e}^{+} \mathrm{e}^{-} \rightarrow \mathrm{hA} \rightarrow \mathrm{b} \overline{\mathrm{b}} \mathrm{b} \overline{\mathrm{b}}$. This corresponds also to limits on $\cos ^{2}(\beta-\alpha)$ in the general extension of the SM with two Higgs boson doublets. The data of the four LEP experiments collected at energies from 88 to $209 \mathrm{GeV}$ are combined. The solid curve is the observed result and the dashed curve shows the expected median. Shaded areas indicate the $1 \sigma$ and $2 \sigma$ probability bands. 
6

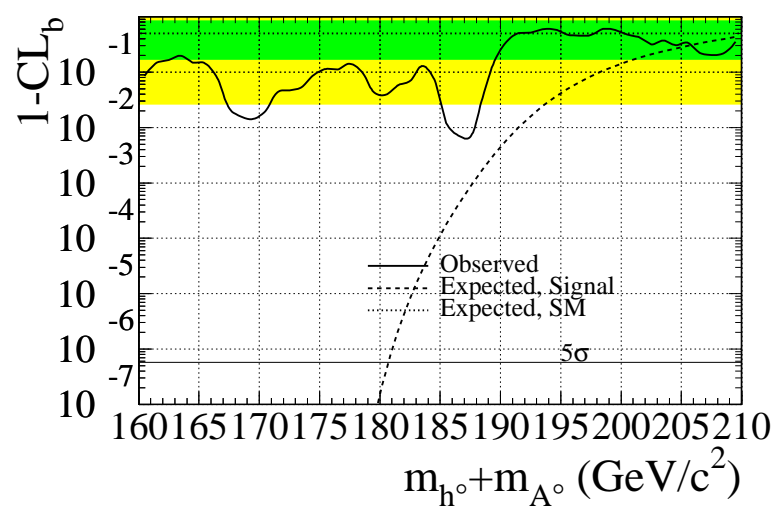

Figure 18. The confidence level $1-C L_{\mathrm{b}}$ as a function of $m_{\mathrm{h}}+m_{\mathrm{A}}$ for the case $m_{\mathrm{h}} \approx m_{\mathrm{A}}$ (where only the $\mathrm{e}^{+} \mathrm{e}^{-} \rightarrow \mathrm{hA}$ process contributes since $\left.\sin ^{2}(\beta-\alpha) \approx 0\right)$. The straight line at 0.5 and the shaded $1 \sigma$ and $2 \sigma$ probability bands represent the expected background-only result. The solid curve is the observed result and the dashed curve shows the expected median for a signal.

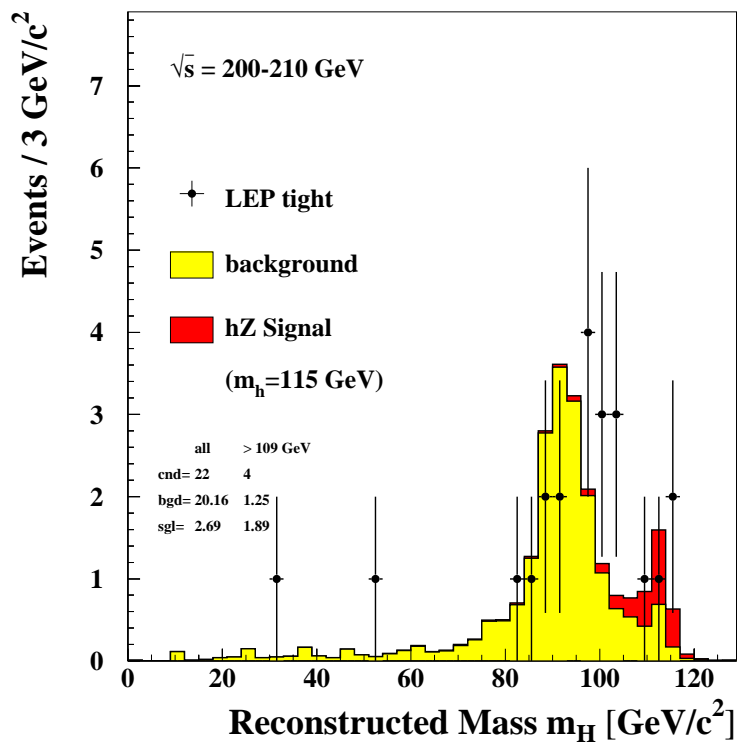

Figure 19. Distribution of the reconstructed SM Higgs boson mass in searches conducted at energies between 200 and $210 \mathrm{GeV}$. The figure displays the data (dots with error bars), the predicted SM background and the prediction for a Higgs boson of $115 \mathrm{GeV}$ mass. The number of data events selected with mass larger than 109 $\mathrm{GeV}$ is 4 , while 1.25 are expected from SM background processes and 1.89 from a $115 \mathrm{GeV}$ signal. Between 96 and $105 \mathrm{GeV} 10$ data events are observed, while 3.6 background events are expected.

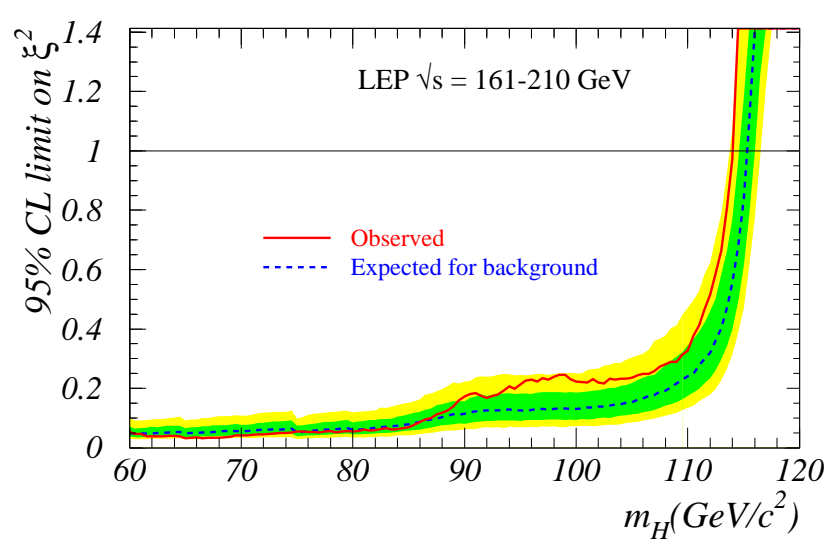

Figure 20. The 95\% CL upper bound on $\xi^{2}$ as a function of $m_{\mathrm{H}}$, where $\xi=g_{\mathrm{HZZ}} / g_{\mathrm{HZZ}}^{\mathrm{SM}}$ is the HZZ coupling relative to the SM coupling. About $2 \sigma$ deviations from the expectation are observed at $m_{\mathrm{H}}=98 \mathrm{GeV}$ and $m_{\mathrm{H}}=115 \mathrm{GeV}$. In the MSSM, hZ production at the lower mass and HZ production at the higher mass are possible.

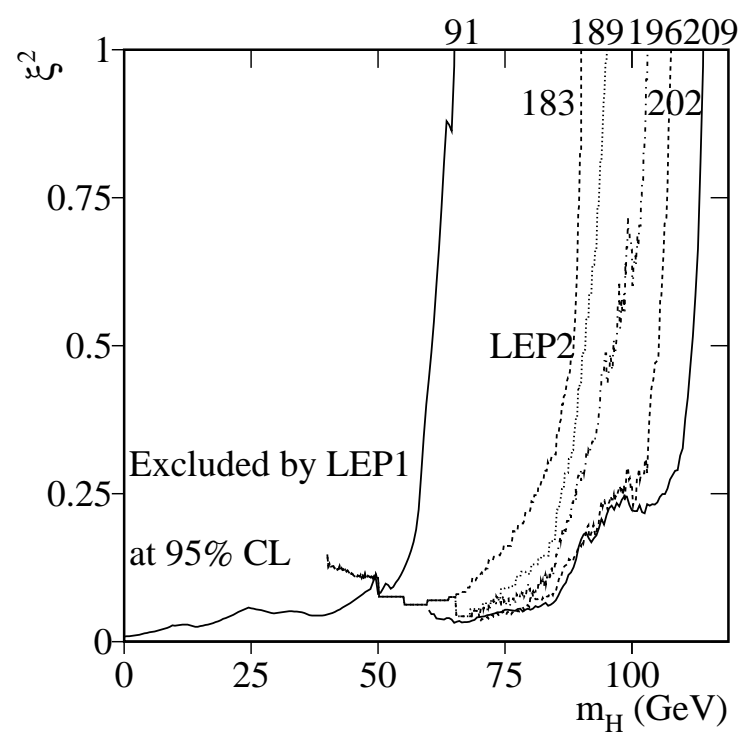

Figure 21. The excluded $\left(\xi^{2}, m_{\mathrm{H}}\right)$ region including $209 \mathrm{GeV}$ data is compared with the results from combined LEP-1 data 19], taken around 91 $\mathrm{GeV}$ center-of-mass energy, and previous LEP-2 limits [14] up to $183,189,196$ and $202 \mathrm{GeV}$. The $\xi^{2}$ limit below $100 \mathrm{GeV}$ does not become significantly stronger when the $209 \mathrm{GeV}$ data, taken in 2000, are included. 


\section{Conclusions}

The LEP-1 and LEP-2 data are consistent with a background-only hypothesis and give stringent mass limits on the neutral Higgs bosons $h$ and A of the MSSM. During the LEP era, MSSM parameter scans reduced - or for early LEP-2 data even removed completely - the benchmark mass limits. The importance of parameter scans is stressed by large parameter regions where the Higgs boson decays invisibly, which is not considered in benchmark results. At the highest center-of-mass energies, benchmark limits are only slightly reduced by a general parameter scan when the results from invisible Higgs boson searches are included. Table 1 compares benchmark and scan mass limits in the MSSM.

The combined LEP data show a preference for the SM Higgs boson of $115.6 \mathrm{GeV}$, which can also be interpreted as a preference for a Higgs boson of that mass in the MSSM. Further small data excesses for Higgs boson pair-production and bremsstrahlung between 90 and $100 \mathrm{GeV}$ allow the hypothesis that $\mathrm{h}, \mathrm{A}$ and $\mathrm{H}$ of the MSSM all have masses below $116 \mathrm{GeV}$. Previously reported MSSM parameter combinations from a general parameter scan for this scenario are supported by the complete data set.

\section{Acknowledgments}

I would like to thank the organizers of the conference for their kind hospitality.

Table 1

Benchmark (b) and scan (s) mass limits in the MSSM. All limits are in GeV at 95\% CL.

\begin{tabular}{|c|c|c|c|c|c|}
\hline$\sqrt{s}(\mathrm{GeV})$ & Data & $m_{\mathrm{h}}^{\mathrm{b}}$ & $m_{\mathrm{A}}^{\mathrm{b}}$ & $m_{\mathrm{h}}^{\mathrm{s}}$ & $m_{\mathrm{A}}^{\mathrm{s}}$ \\
\hline 91 [5]:6] & L3 & 41.0 & none & 25 & none \\
\hline 172 [6] & DELPHI & 59.5 & 51.0 & 30 & none \\
\hline 183 20,21 & DELPHI & 74.4 & 75.2 & 67 & 75 \\
\hline $189[9]$ & OPAL & 74.8 & 76.5 & 72 & 76 \\
\hline 189 [11, 10 & DELPHI & 82.6 & 84.1 & 75 & 78 \\
\hline 202 & DELPHI & 85.9 & 86.5 & 85 & 86 \\
\hline 202 13, 14 & LEP & 88.3 & 88.4 & 86 & 87 \\
\hline 209 15,4] & DELPHI & 89.6 & 90.7 & 89 & 89 \\
\hline
\end{tabular}

\section{REFERENCES}

1. A. Sopczak, Phys. Rep., Higgs Physics at LEP-1, in press.

2. A. Sopczak, 'Complete LEP Data: Status of Higgs Boson Searches', NANP-1, Moscow, June 2001, proc. in press, hep-ph/0112082.

3. ALEPH, DELPHI, L3 and OPAL Coll. and the LEP working group for Higgs boson searches, LHWG Note/2001-04.

4. DELPHI Coll., DELPHI 2001-81 CONF-509, EPS HEP 2001 (Budapest) and LP01 (Rome).

5. A. Sopczak, Ph.D. Thesis, Univ. of California, San Diego (1992);

L3 Coll., Phys. Lett. B 294 (1992) 457;

Z. Phys. C 57 (1993) 355.

6. J. Rosiek and A. Sopczak, Phys. Lett. B 341 (1995) 419.

7. DELPHI Coll., Eur. Phys. J. C 2 (1998) 1.

8. A. Sopczak, Eur. Phys. J. C 9 (1999) 107.

9. OPAL Coll., Eur. Phys. J. C 12 (2000) 567.

10. DELPHI Coll., Eur. Phys. J. C 17 (2000) 549.

11. DELPHI Coll., Eur. Phys. J. C 17 (2000) 187.

12. DELPHI Coll., subm. Eur. Phys. J. C, CERN-EP/2001-87.

13. ALEPH, DELPHI, L3 and OPAL Coll. and the LEP working group for Higgs boson searches, ALEPH 2000-074 CONF 2000-051, DELPHI 2000-148 CONF-447, L3 Note 2600, OPAL TN 661.

14. A. Sopczak, hep-ph/0004015, PASCOS-99, (World Scientific, Singapore, 2000), p. 511.

15. DELPHI Coll., DELPHI 2001-78 CONF-506, EPS HEP 2001 (Budapest) and LP01 (Rome).

16. DELPHI Coll., DELPHI 2001-79 CONF-507, EPS HEP 2001 (Budapest) and LP01 (Rome).

17. A. Sopczak, hep-ph/0011285, Proc. DPF2000, Columbus, Ohio, Aug. 9-12, 2000, http://www.dpf2000.org/BSM1.htm\#ses4.

18. ALEPH, DELPHI, L3 and OPAL Coll. and the LEP working group for Higgs boson searches, CERN-EP/2001-55.

19. A. Sopczak, DESY 97-129, Indian J. Phys. A 72 (1998) 495.

20. DELPHI Coll., DELPHI 98-95 CONF-163, ICHEP 1998 (Vancouver).

21. DELPHI Coll., DELPHI 98-124 CONF-185, ICHEP 1998 (Vancouver). 